\title{
Localization of the Chain Recurrent set using Shape theory and Symbolical Dynamics
}

https://doi.org/10.1515/taa-2019-0002

Received December 26, 2018; accepted June 14, 2019

\begin{abstract}
The main aim of this paper is localization of the chain recurrent set in shape theoretical framework.Namely, using the intrinsic approach to shape from [1] we present a result which claims that under certain conditions the chain recurrent set preserves local shape properties. We proved this result in [2] using the notion of a proper covering. Here we give a new proof using the Lebesque number for a covering and verify this result by investigating the symbolical image of a couple of systems of differential equations following [3].
\end{abstract}

Keywords: chain recurrent set, shape, intrinsic shape, symbolical image, attractor, Morse decomposition, Lyapunov function

MSC: 54H20, 54C56, 37B20, 37B25

Several authors have pointed out the connections between shape theory and topological dynamics based on the approach to shape developed by Borsuk. When dealing with spaces with complicated local behaviour, which often occurs in dynamical framework, shape theory turns out to be an appropriate tool.The use of shape theory in the study of dynamical systems was initiated by Hastings in [4].Other authors have shown how to apply shape theory to obtain global properties of attractors in the papers [5], [6], [7], [8]. Recently, using the intrinsic shape (the approach to shape without use of external spaces) [1], [9], several results are obtained showing the advantages of intrinsic approach to shape in some situations. Applying intrinsic shape in dynamical systems seems very natural. The most natural application of intrinsic shape is for investigation of objects that appear in dynamical systems, like attractors, limit sets or various types of recurrent sets.The papers [2] and [10] are good examples of that.

In the paper [11] a weak form of recurrence called chain recurrence is introduced by Conley. When we make computer simulation, calculating orbit of a point in each step we have rounding errors, so each time we obtain pseudo-orbit ( $\epsilon$-chain) instead of true orbit of observed point. So we will rather detect chain recurrent points than periodic orbits in computer experiments.

Chain recurrent set of continuous dynamical systems on compact metric spaces has many interesting properties. It is closed, flow invariant and restriction of the flow to chain recurrent set does not change chain recurrent points.It is also well known that flow restricted to limit set of any point in $X$ is chain recurrent.

In this paper we give a theorem which shows that under certain conditions the shape of the chain recurrent set is locally invariant. An investigation of the symbolic image gives an opportunity to get a neighborhood of the chain recurrent set and using the algorithm for localizing neighborhoods provided by Osipenko in [3] we obtained a programming code which was successfully tested on a couple of systems of differential equations. In what follows we assume that the phase space $X$ in which all dynamics take place is always a compact metric space.

`Corresponding Author: M. Shoptrajanov: University of Ss. "Cyril and Methodius", Faculty of Natural Sciences and Mathematics, Institute of Mathematics, Skopje, Republic of North Macedonia, E-mail: martin@pmf.ukim.mk 


\section{Intrinsic Shape}

The first paper about intrinsic shape is [12] where a shape morphism between compact metric spaces has been defined for the first time using the notion of $\mathcal{V}$-continuity.

We will give a brief construction of the shape category using the intrinsic approach from [1]. The approach follows the main steps of construction of homotopy theory.

Instead of $f, g: X \rightarrow Y$ continuous functions we take $\left(f_{n}\right),\left(g_{n}\right): X \rightarrow Y$ proximate sequences of functions and define a relation of homotopy $\left(f_{n}\right) \simeq\left(g_{n}\right)$.

Like in homotopy theory where $X$ and $Y$ have the same homotopy type if there exist a continuous function $f: X \rightarrow Y$ with a homotopy inverse, $X$ and $Y$ have the same (intrinsic) shape if there exist a proximate sequence $\left(f_{n}\right): X \rightarrow Y$ with a homotopy inverse.

Let's start with some of the basic definitions: For collections $\mathcal{U}$ and $\mathcal{V}$ of subsets of $X$, $U \prec \mathcal{V}$ means that $\mathcal{U}$ refines $\mathcal{V}$, i.e., each $U \in \mathcal{U}$ is contained in some $V \in \mathcal{V}$. By covering we understand a covering consisting of open sets.

Definition 1.1. Let $X, Y$ be compact metric spaces, and $\mathcal{V}$ be a finite covering of $Y$. The function is $\mathcal{V}$-continuous, if for any $x \in X$, there exists a neighborhood $U$ of $x$, such that $f(U) \subseteq V$, for some member $V \in \mathcal{V}$.

(The family of all $U$, form a covering of $X$, and since $X$ is compact there is a finite subcovering. Shortly, we say that $f: X \rightarrow Y$ is $\mathcal{V}$-continuous, if there exists a finite covering $\mathcal{U}$ such that $f(\mathcal{U}) \prec \mathcal{V})$.

Let $\mathcal{V}$ is a finite covering of $Y$ and $V \in \mathcal{V}$. The open set $\operatorname{st}(V)$ (star of $V$ ) is the union of all $W \in \mathcal{V}$ such that $W \cap V \neq \emptyset$. We form a new covering of $Y, \operatorname{st}(\mathcal{V})=\{\operatorname{st}(V) \mid V \in \mathcal{V}\}$.

Definition 1.2. The functions $f, g: X \rightarrow Y$ are $\mathcal{V}$-homotopic, if there exists a function $F: X \times I \rightarrow Y$ such that:

i) $F: X \times I \rightarrow Y$ is $\operatorname{st}(\mathcal{V})$ - continuous,

ii) $F: X \times I \rightarrow Y$ is $\mathcal{V}$-continuous at all points of $X \times \partial I$

iii) $F(x, 0)=f(x), F(x, 1)=g(x)$.

The relation of homotopy is an equivalence relation and is denoted by $f \stackrel{\mathcal{V}}{\simeq} g$

The main notion for intrinsic definition of shape for compact metric spaces is the notion of proximate sequence.

Definition 1.3. The sequence $\left(f_{n}\right)$ of functions $f_{n}: X \rightarrow Y$ is a proximate sequence from $X$ to $Y$, if for some sequence $V_{1} \succ V_{2} \succ \ldots$ cofinal in the set of finite coverings, for all indices $m \geq n, f_{n}$ and $f_{m}$ are homotopic as $\mathcal{V}_{n}$ - continuous functions (cofinal means that for any finite covering $\mathcal{V}$ there exists $\mathcal{V}_{n}$ such that $\mathcal{V}_{n} \prec \mathcal{V}$ ). In this case we say that $\left(f_{n}\right)$ is a proximate sequence over $\left(\mathcal{V}_{n}\right)$.

We mention that if $\left(f_{n}\right)$ and $\left(f_{n}^{\prime}\right)$ are proximate sequences from $X$ to $Y$, then there exists a sequence $\left(\mathcal{V}_{n}\right)$ of finite coverings, such that $\left(f_{n}\right)$ and $\left(f_{n}^{\prime}\right)$ are proximate sequences over $\left(\mathcal{V}_{n}\right)$.

Two proximate sequences $\left(f_{n}\right),\left(f_{n}^{\prime}\right)$ are homotopic if for some sequence $\mathcal{V}_{1} \succ V_{2} \succ \ldots$ cofinal in the set of finite coverings, $\left(f_{n}\right)$ and $\left(f_{n}^{\prime}\right)$ are proximate sequences over $\left(\mathcal{V}_{n}\right)$, and for all integers $n, f_{n}$ and $f_{n}^{\prime}$ are homotopic as $\mathcal{V}_{n}$ - continuous functions.

This is an equivalence relation and we denote $\left(f_{n}\right) \simeq\left(f_{n}^{\prime}\right)$. The homotopy class is denoted by $\left[\left(f_{n}\right)\right]$.

Let $\left(f_{n}\right): X \rightarrow Y$ be proximate sequence over $\left(\mathcal{V}_{n}\right)$, and let $\left(g_{n}\right): Y \rightarrow Z$ be a proximate sequence over $\left(\mathcal{W}_{n}\right)$.For a covering $\mathcal{W}_{n}$ of $Z$, there exist a covering $\mathcal{V}_{k_{n}}$ of $Y$ such that $g\left(\mathcal{V}_{k_{n}}\right) \prec \mathcal{W}_{n}$. Then the composition of these two proximate sequences is the proximate sequence $\left(h_{n}\right)=\left(g_{n} f_{k_{n}}\right): X \rightarrow Z$. This proximate sequence is unique up to homotopy.

Compact metric spaces and homotopy classes of proximate sequences form the category whose isomorphisms induce classification which coincide with the standard shape classification, i.e., isomorphic spaces in this category have the same shape. 


\section{Basic notions and theorems about dynamical systems}

One approach to dynamical systems introduced by Poincare and followed by Morse and Conley can be described as follows: First of all a finite family of invariant sets $\left\{M_{i}\right\}_{i=1}^{n}$ is located such that the flow in the complement set $X \backslash \bigcup_{j=1}^{n} M_{j}$ is fairly simple. Then some kind of local analysis is performed around those sets( local means that only the flow in arbitrary small neighborhoods is involved). Finally this local information is put together in some way involving the global topology of the phase space. Trivial examples of such sets are finitely many critical points $p_{1}, p_{2}, \ldots, p_{n}$ which contain all limit sets on a compact manifold. This approach leads to introduction of a Morse decomposition.

Before introducing the concept of these sets we shall give a brief review of the basic notions from dynamical system theory.

A flow in a metric space $(X, d)$ is a continuous map $\varphi: X \times \mathbb{R} \rightarrow X$ satisfying the following two condition: i) $\varphi(x, 0)=x$ for all $x \in X$

ii) $\varphi(\varphi(x, t), s)=\varphi(x, t+s)$ for all $x \in X$ and $t, s \in \mathbb{R}$

If we replace the set $\mathbb{R}$ with $\mathbb{R}^{+}$we get the corresponding notion of semi-dynamical system. The map $\varphi$ is called a phase map, and the corresponding space $X$ a phase space.

One of the important problems in dynamical systems concerns the asymptotic behavior of trajectories as time goes to plus or minus infinity. Limit sets are fundamental tools for this problem.

Positive limit set for arbitrary subset $N \subseteq X$ is the set:

$$
\omega(N)=\left\{x \in X \mid \exists x_{n} \in N, t_{n} \rightarrow \infty, \varphi\left(x_{n}, t_{n}\right) \rightarrow x\right\}
$$

Analogously, negative limit set for arbitrary subset $N \subseteq X$ is the set:

$$
\alpha(N)=\left\{x \in X \mid \exists x_{n} \in N, t_{n} \rightarrow-\infty, \varphi\left(x_{n}, t_{n}\right) \rightarrow x\right\}
$$

A set $M$ is invariant if $\varphi(M, t) \subseteq M$, for all $t \in \mathbb{R}$. If we replace the set $\mathbb{R}$ with $\mathbb{R}^{+}$or $\mathbb{R}^{-}$, we get the corresponding notion of positive and negative invariance. If $\varphi(x, t)=x$, for all $t \in \mathbb{R}$, the point $x$ is called rest point.

A set $M$ is stable if every neighborhood $U$ of $M$ admits a positively invariant neighborhood $V$ of $M$ such that $V \subseteq U$.

A compact invariant set $Y \subseteq X$ is called an attractor if it admits a neighborhood $U$ such that $\omega(U)=Y$.

Analogously, a compact invariant set $Y \subseteq X$ is called an repellor if it admits a neighborhood $U$ such that $\alpha(U)=Y$.

It is known that every attractor is a stable set (for example [13]).

Definition 2.1. Let $K=\left\{K_{j} \mid j \in J\right\}$ be a family of disjoint compact invariant subsets of the phase space X. A Lyapunov function for $K$ is a continuous function $\tau: X \rightarrow \mathbb{R}$ such that:

i) $\tau(\varphi(x, t))<\tau(x), \forall t>0, \forall x \notin \bigcup_{j \in J} K_{j}$

ii) $\tau\left(K_{j}\right)=c_{j}, \forall j \in J,\left(c_{j} \neq c_{i}\right.$ for $\left.i \neq j\right)$.

Definition 2.2. Let $\varphi$ be a continuous flow on a metric space $X$. An isolated invariant set is a subset $S \subseteq X$ which is the maximal invariant set in a compact neighborhood of itself. Such a neighborhood is called an isolating neighborhood.

Definition 2.3. Morse decomposition for $S$ is a collection $\left\{M_{i}\right\}_{i=1}^{n}$, where each $M_{i}$ is an isolated invariant subset of $S$ and for all $x \in S \backslash \bigcup_{j=1}^{n} M_{j}$, there exist $i, j \in\{1,2, \ldots, n\}$ such that $i>j, \alpha(x) \subseteq M_{i}$ and $\omega(x) \subseteq M_{j}$. 


\section{Example 2.1.}

Consider the following differential equation on the compact interval $X=[0,3]$ :

$$
\dot{x}=x(x-1)(x-2)^{2}(x-3)
$$

This system has the following Morse decompositions:

$\{\{0\},[1,3]\},\{\{0\},\{1\},[2,3]\},\{\{0\},\{3\},[1,2]\}$ and others. Obviously it is not unique. But there is a unique finest Morse decomposition $\{\{0\},\{1\},\{2\},\{3\}\}$.

By decomposing Morse sets into attractor-repellor pairs, Morse decompositions can be refined. While many systems have a finest Morse decomposition, there are systems which have (countably) infinitely many attractor-repellor pairs and hence no finest Morse decomposition. The process of extracting all attractorrepellor pairs leads to the chain recurrent set $\mathcal{E R}(\varphi)$ introduced by Conley [14].

Definition 2.4. Let $(x, y) \in X \times X$ and $\varepsilon>0, t>0$. $(\varepsilon, t, \varphi)$-chain from $x$ to $y$ is a collection $\{x=$ $\left.x_{1}, x_{2}, \ldots, x_{n}, x_{n+1}=y ; t_{1}, t_{2}, \ldots, t_{n}\right\}$ such that for all $i \in\{1,2, \ldots, n\}, t_{i} \geq t$ and $d\left(\varphi\left(x_{i}, t_{i}\right), x_{i+1}\right)<\varepsilon$.

$$
P(\varphi)=\{(x, y) \mid \forall \varepsilon, t>0 \text {, there exists }(\varepsilon, t, \varphi) \text {-chain from } x \text { to } y\} \text {. }
$$

$\operatorname{Now} \mathcal{C R}(\varphi)=\{x \mid(x, x) \in P(\varphi)\}$

\section{Example 2.2.}

Consider the complete metric space $S^{1}$, the 1 - dimensional sphere, which we identify here with $\mathbb{R} / 2 \pi$. On $S^{1}$ the differential equation $\dot{x}=\sin ^{2}(x)$ defines a dynamical system. In this case we have $\operatorname{eR}(\varphi)=S^{1}$ which is easily proved.

The following lemma is proved in [2]

Lemma 2.1. Arbitrary Morse decomposition $\left\{M_{i=1}^{n}\right\}$ for a compact metric space $(X, d)$ with a flow $\varphi$, admits a Lyapunov function.

From [15] are the following two results: 1) Every two element Morse decomposition $\left\{M_{1}, M_{2}\right\}$ is in fact an attractor-repellor pair 2) For arbitrary Morse decomposition $M=\left\{M_{1}, M_{2}, M_{3}, \ldots \ldots, M_{n}\right\}$ the first member $M_{1}$ is an attractor and the last one $M_{n}$ is a repellor.

The next result is proved in [8].

Theorem 2.1. Every global attractor $A$ of a semi-dynamical system defined on a compact metric space $X$ has the same shape as the phase space, i.e. $\operatorname{Sh}(A)=\operatorname{Sh}(X)$.

Using the results previously stated we proved in [2] also the following theorem:

Theorem 2.2. Every two element Morse decomposition $\left\{M_{1}, M_{2}\right\}$ has corresponding compact neighborhoods $U_{1}, U_{2}$ such that $\operatorname{Sh}\left(U_{i}\right)=\operatorname{Sh}\left(M_{i}\right), i=1,2$.

In the next section we shall prove a more general form of this theorem using the following result also known as Keesling's reformulation of Beck's theorem, [16]. 
Theorem 2.3. Let $X$ be a metric space and let $\varphi: X \times R \rightarrow X$ be a flow on $X$ with $S$ as set of fixed points.Then for any closed set $S^{\prime}$ containing $S$ one can construct a new flow $\varphi^{\prime}: X \times R \rightarrow X$ with $S^{\prime}$ as set of fixed points. Moreover, for any $x \in X \backslash S^{\prime}$ with trajectory $o(x)$ under $\varphi$, the trajectory of $x$ under $\varphi^{\prime}$ is just the set of points which can be joined to $x$ by an arc in $o(x) \backslash S^{\prime}$.

\section{Shape of the Chain Recurrent Set}

In the paper [2] we introduced the notion of a proper covering $\mathcal{V}$ and defined a retraction $r_{\mathcal{V}}$ adjoint to $\mathcal{V}$. Also we invoked previous results from theory of retracts and Non-continuous Topology in order to answer the question that is naturally imposed by theorem 2.2 about the shape of members of a Morse decomposition with more than two elements. Here we give a new proof only using the notion of a Lebesque number for a covering which enables us to state the claim about the local shape properties of the chain recurrent set $\mathcal{C R}(\varphi)$. We use the following notation: $T(x, \varepsilon)$-open ball centered at $x$ and radius $\varepsilon, S(x, \varepsilon)$ sphere centered at $x$ and radius $\varepsilon$.

Theorem 3.1. Let $(X, d)$ be a compact metric space with a flow $\varphi$. For arbitrary Morse decomposition for $X$, which admits Lyapunov function $\tau: X \rightarrow \mathbb{R}$ such that $\tau^{-1}\left(c_{i}\right)=M_{i}$, there exist compact neighborhoods $U_{i}$ of $M_{i}$,such that $\operatorname{Sh}\left(U_{i}\right)=\operatorname{Sh}\left(M_{i}\right)$.

Proof. First let us note that we can suppose that the flow on the set $\bigcup_{i=1}^{n} M_{i}$ is stationary. Namely, if this is not the case then using Theorem 2.3 with the choice $S^{\prime}=\bigcup_{i=1}^{n} M_{i}$ we will get a new flow in which this is the case. Also let us emphasize that $M=\left\{M_{1}, M_{2}, M_{3}, \ldots, M_{n}\right\}$ is also a Morse decomposition for the new flow. It is enough to consider sets $M_{i}$ such that $2 \leq i \leq(n-1)$, since the result about attractor and repelor is already proved. So let us pick arbitrary set $M_{i}, 2 \leq i \leq(n-1)$. We denote $\tau\left(M_{i}\right)=c_{i}$, and form the sets $U^{+}=\tau^{-1}\left[c_{i}, c_{i}+\varepsilon\right]$ and $U^{-}=\tau^{-1}\left[c_{i}-\varepsilon, c_{i}\right]$, where $\epsilon>0$ is sufficiently small such that the following holds:

$$
\left(M_{1} \cup M_{2} \cup \ldots M_{i-1} \cup M_{i+1} \cup \ldots \cup M_{n}\right) \bigcap\left(U^{+} \cup U^{-}\right)=\emptyset
$$

Now let's consider the semi-dynamical systems $\left(U^{+}, \varphi_{\mid U^{+}}\right)$and $\left(U^{-}, \varphi_{\mid U^{-}}\right)$. This is possible because the sets $U^{+}=\tau^{-1}\left[c_{i}, c_{i}+\varepsilon\right]$ and $U^{-}=\tau^{-1}\left[c_{i}-\varepsilon, c_{i}\right]$ are positively and negatively invariant, respectively. We will show this assumption.

Namely, if $x \in U^{+}=\tau^{-1}\left[c_{i}, c_{i}+\varepsilon\right]$ then $\tau(x) \in\left[c_{i}, c_{i}+\epsilon\right]$, so for arbitrary $t>0$ using the property of Lyapunov function, we have $\tau(\varphi(x, t))<\tau(x) \leq c_{i}+\epsilon$. Of course the possibility $x \in M_{i}$ is trivial so we assume that $x \notin M_{i}$. Let us assume that $\tau(\varphi(x, t))<c_{i}$. Then using continuity of $\varphi$ and $\tau$ from $\tau(\varphi(x, t))<c_{i}<\tau(x)$ we get that a real number $t^{\star}>0$ exists such that $\tau\left(\varphi\left(x, t^{\star}\right)\right)=c_{i}$ which means that $\varphi\left(x, t^{\star}\right) \in M_{i}$. Now using the fact that $M_{i}$ is invariant, we get $x \in M_{i}$, which is a contradiction. So $\tau(\varphi(x, t)) \geq c_{i}$, from where we get that $\varphi(x, t) \in U^{+}$. Similarly, $U^{-}$is negatively invariant. Now we shell prove that for the first semidynamical system $\left(U^{+}, \varphi_{\mid U^{+}}\right) M_{i}$ is an attractor, i.e. $\omega\left(U^{+}\right)=M_{i}$, and for the second one $\left(U^{-}, \varphi_{\mid U^{-}}\right)$a repelor, i.e. $\alpha\left(U^{-}\right)=M_{i}$. Namely, for the first one it is clear that $\omega\left(U^{+}\right) \supseteq M_{i}$. If we assume that $z \in \omega\left(U^{+}\right)$then from the invariance of the limit set $\omega\left(U^{+}\right)$as well as from $\omega\left(U^{+}\right) \subseteq U^{+}$, we have that $\varphi(z, R) \subseteq U^{+}$which means that $\alpha(z) \subseteq U^{+}, \omega(z) \subseteq U^{+}$, but from the fact that all limit sets of points are contained in $\bigcup_{i=1}^{n} M_{i}$, we get that $\alpha(z) \cup \omega(z) \subseteq M_{i}$ from where we have that $z \in M_{i}$. The proof for the second semi-dynamical system is similar.

Now it easily follows from definitions of limit sets that for arbitrary $\epsilon>0, \exists t_{0} \in \mathbb{R}^{+}$such that $\forall t \geq t_{0}$, $\forall u \in U^{+}, \varphi(u, t) \in T\left(M_{i}, \varepsilon\right)$. Similarly, we have that for every $\epsilon>0, \exists t_{0} \in \mathbb{R}^{-}$such that $\forall t \leq t_{0}, \forall u \in$ $U^{-}, \varphi(u, t) \in T\left(M_{i}, \varepsilon\right)$.

We are ready to define a map $a:\left(U^{+} \cup U^{-}\right) \times \mathbb{R}^{+} \rightarrow M_{i}$ in the following way: For points $(x, t) \in U^{+} \times \mathbb{R}^{+}$, we go with the flow until the point $\varphi(x, t)$, then we measure the distance $d\left(\varphi(x, t), M_{i}\right)$ which by compactness of the set $M_{i}$ is achieved in some point $m_{x}^{t} \in M_{i}$, that is $d\left(\varphi(x, t), M_{i}\right)=d\left(\varphi(x, t), m_{x}^{t}\right)$. Of course this point may not be unique but never the less we can pick any such point. So we define $a(x, t)=m_{x}^{t}$, for $(x, t) \in U^{+} \times \mathbb{R}^{+}$. For points $(x, t) \in U^{-} \times \mathbb{R}^{+}:$we go with the flow until the point $\varphi(x,-t)$, then we measure 
the distance $d\left(\varphi(x,-t), M_{i}\right)$ which by compactness of the set $M_{i}$ is achieved in some point $m_{x}^{t} \in M_{i}$, that is $d\left(\varphi(x,-t), M_{i}\right)=d\left(\varphi(x,-t), m_{x}^{t}\right)$. Of course this point may not be unique but never the less we can pick any such point. So we define $a(x, t)=m_{x}^{t}$, for $(x, t) \in U^{-} \times \mathbb{R}^{+}$.

Let us note that this map is well defined on $M_{i} \times \mathbb{R}^{+}$because the following holds: $a(x, t)=\varphi(x, t)=x=$ $\varphi(x,-t), \forall(x, t) \in M_{i} \times \mathbb{R}^{+}$, of course having in mind that the flow on $M_{i}$ is stationary.

Let $\left(V_{n}\right)$ be a cofinal sequence of coverings for $M_{i}$. We shall define a sequence of real numbers $t_{n} \in \mathbb{R}^{+}$, such that the maps $f_{n}: U \rightarrow M_{i}$ defined by $f_{n}(x)=a\left(x, t_{n}\right)$ are $\mathcal{V}_{n}$-continuous.

Construction of the sequence $\left(t_{n}\right)$ : We choose $\varepsilon=\frac{\lambda_{n}}{8}$ where $\lambda_{n} \leq 1$ is the Lebesque number of the covering $\mathcal{V}_{n}$. There exist strictly monotonically increasing sequences $t_{n}^{+}, t_{n}^{-} \longrightarrow \infty$ such that the following conditions are satisfied:

$$
\varphi\left(u, t_{n}^{+}\right) \in T\left(M_{i}, \frac{\lambda_{n}}{8}\right), \forall u \in \mathcal{U}^{+} ; \varphi\left(u,-t_{n}^{-}\right) \in T\left(M_{i}, \frac{\lambda_{n}}{8}\right), \forall u \in \mathcal{U}^{-}
$$

. Let $\alpha_{n}=\min \left\{\tau(x) \mid x \in S\left(M_{i}, \lambda_{n} / 8\right) \cap \mathcal{U}^{+}\right\}, \beta_{n}=\max \left\{\tau(x) \mid x \in S\left(M_{i}, \lambda_{n} / 8\right) \cap \mathcal{U}^{-}\right\}$. We will choose the time sequences in such a way to satisfy the following inequality, namely $t_{n}^{-} \geq \frac{c_{i}-\beta_{n}}{\alpha_{n}-c_{i}} \cdot t_{n}^{+}$. Finally we make the choice for $t_{n}=\frac{t_{n}^{-}}{c_{i}-\beta_{n}}\left(t_{n} \geq t_{n}^{+}, t_{n}^{-}\right.$for sufficiently large $\left.n\right)$.

Lemma 3.1. The maps $f_{n}, f_{m}: \mathcal{U} \longrightarrow M_{i}$, for $m \geq n$ are $\mathcal{V}_{n}$-homotopic.

Proof. We define the following map $H_{n}: U \times I \longrightarrow M_{i}$ by:

$$
H_{n}(x, t)=a\left(x,(1-t) t_{n}+t t_{m}\right) .
$$

Let us note that the connecting relations are obvious. We shall prove that $H_{n}$ is $V_{n}$-continuous homotopy.

Let $x_{0} \in \operatorname{int} M_{i}, t_{0} \in I$. The flow is stationary on $M_{i}$ so we get $H_{n}\left(x_{0}, t_{0}\right)=x_{0}$. There exists neighborhood $W_{0}$ of $x_{0}$ such that $W_{0} \subseteq M_{i}$ and $W^{0}$ of $t_{0}$ such that $H_{n}(x, t)=x$, for all $x \in W_{0}, t \in W^{0}$. But this means that $H_{n}$ is continuous in $\left(x_{0}, t_{0}\right)$, so is $V_{n}$ as well.

Now, let $x_{0} \in \partial M_{i}, t_{0} \in I$. Let us note that $h_{n, m}(t)=(1-t) t_{n}+t t_{n} \geq t_{n}$. For the points $x \in \mathcal{U}^{+} \backslash M_{i}, t \in I$ is valid: $d\left(a\left(x, h_{n, m}(t), \varphi\left(x, h_{n, m}(t)\right)<\frac{\lambda_{n}}{8}\right.\right.$. Let $\varepsilon<\frac{3 \lambda_{n}}{8}$. There exists neighborhood $W_{1}$ of $x_{0} \in \partial M_{i}$ and $W^{1}$ of $t_{0} \in I$ such that $d\left(\varphi\left(x, h_{n, m}(t)\right), \varphi\left(x_{0}, h_{n, m}\left(t_{0}\right)\right)\right)<\varepsilon$, for all $x \in W_{1}, t \in W^{1}$. Now, for $x \in W_{1} \cap\left(\mathcal{U}^{+} \backslash M_{i}\right)$, $t \in W^{1}:$

$$
\begin{gathered}
d\left(a\left(x, h_{n, m}(t)\right), \varphi\left(x_{0}, h_{n, m}\left(t_{0}\right)\right)\right) \leq d\left(a\left(x, h_{n, m}(t)\right), \varphi\left(x, h_{n, m}(t)\right)\right) \\
+d\left(\varphi\left(x, h_{n, m}(t)\right), \varphi\left(x_{0}, h_{n, m}\left(t_{0}\right)\right)\right)<\frac{\lambda_{n}}{2} .
\end{gathered}
$$

Now $H_{n}\left(W_{1} \cap \mathcal{U}^{+} \backslash M_{i}, W^{1}\right) \subseteq T\left(H_{n}\left(x_{0}, t_{0}\right), \lambda_{n} / 2\right)$, which means that there exists $V_{n} \in V_{n}$ such that $H_{n}\left(W_{1} \cap U^{+} \backslash M_{i}, W^{1}\right) \subseteq V_{n}$. Analogously, there exists neighborhoods $W_{2}$ of $x_{0}$ and $W^{2}$ of $t_{0}$ such that $H_{n}\left(W_{2} \cap \mathcal{U}^{-} \backslash M_{i}, W^{2}\right) \subseteq T\left(H_{n}\left(x_{0}, t_{0}\right), \lambda_{n} / 2\right)$, so for the same $V_{n} \in \mathcal{V}_{n}$ is valid $H_{n}\left(W_{2} \cap \mathcal{U}^{-} \backslash M_{i}, W^{2}\right) \subseteq V_{n}$. Moreover, let us note that there exists neighborhoods $W_{3}$ of $x_{0}$ and $W^{3}$ of $t_{0}$ such that the following inclusion is valid: $H_{n}\left(W_{3} \cap M_{i}, W^{3}\right) \subseteq V_{n}$. Finally we choose neighborhood $W_{1} \cap W_{2} \cap W_{3}$ for $x_{0}$ and neighborhood $W^{1} \cap W^{2} \cap W^{3}$ for $t_{0}$. Hence

$$
H_{n}\left(W_{1} \cap W_{2} \cap W_{3}, W^{1} \cap W^{2} \cap W^{3}\right) \subseteq V_{n} .
$$

Now, we shall discuss the points $x_{0} \in \mathcal{U}^{+} \backslash M_{i}, t_{0} \in I$. First let us note that $H_{n}\left(x_{0}, t_{0}\right)=a\left(x_{0},\left(1-t_{0}\right) t_{n}+\right.$ $\left.t_{0} t_{n}\right)$. Now, for arbitrary $\varepsilon>0$ there exists neighborhood $W_{0}$ of $x_{0}$ and $W^{0}$ of $t_{0}$ such that $d\left(\varphi\left(x, h_{n, m}(t)\right), \varphi\left(x_{0}, h_{n, m}\left(t_{0}\right)\right)\right)$ $\varepsilon$, for all $x \in W_{0}, t \in W^{0}$, where $h_{n, m}(t)=(1-t) t_{n}+t t_{n}$. Again, having in mind that $h_{n, m}(t) \geq t_{n}$, we obtain the following estimates:

$$
\begin{gathered}
d\left(a\left(x, h_{n, m}(t)\right), a\left(x_{0}, h_{n, m}\left(t_{0}\right)\right)\right) \leq d\left(a\left(x, h_{n, m}(t)\right), \varphi\left(x, h_{n, m}(t)\right)\right) \\
+d\left(\varphi\left(x, h_{n, m}(t)\right), \varphi\left(x_{0}, h_{n, m}\left(t_{0}\right)\right)\right)+d\left(\varphi\left(x_{0}, h_{n, m}\left(t_{0}\right)\right), a\left(x_{0}, h_{n, m}\left(t_{0}\right)\right)\right)<\frac{\lambda_{n}}{8}+\varepsilon+\frac{\lambda_{n}}{8},
\end{gathered}
$$


for all $x \in W_{0}, t \in W^{0}$. Now, making the choice for $\varepsilon<\lambda_{n} / 4$, we obtain that $H_{n}\left(W_{0}, W^{0}\right) \subseteq T\left(H_{n}\left(x_{0}, t_{0}\right), \lambda_{n} / 2\right)$. Hence, there exists $V_{n} \in \mathcal{V}_{n}$ such that $H_{n}\left(V_{0}, V^{0}\right) \subseteq V_{n}$.

The case for the point $x_{0} \in \mathcal{U}^{-} \backslash M_{i}, t_{0} \in I$, is analogous to the previous discussion.

According to the previous lemma, we can confirm that there exists a shape morphism $\left[\left(f_{n}\right)\right]: \mathcal{U} \longrightarrow M_{i}$.

Proposition 3.1. The shape morphism $\left[\left(f_{n}\right)\right]$ is a shape deformation retract. Consequently $\operatorname{Sh}\left(M_{i}\right)=\operatorname{Sh}\left(U_{i}\right)$, $i=1,2, \ldots, n$.

Proof. Namely, let us note that it is enough to prove that the following composition is valid $\left[\left(i_{n}\right)\right] \circ\left[\left(f_{n}\right)\right]=$ $\left[\left(1_{\mathcal{U}}\right)\right]$, where $\left[\left(i_{n}\right)\right]$ is the shape morphism induced by the $i=i_{n}: M_{i} \longrightarrow \mathcal{U}$. Let $\left(\mathcal{V}_{n}\right)$ be a cofinal sequence of coverings of $\mathcal{U}$ such that $\left(f_{n}\right)$ is proximate sequence over $\left(\mathcal{V}_{n} \cap M_{i}\right)$ with Lebesque number $\lambda_{n}^{\prime} \leq \lambda_{n}$. $\left(\lambda_{n}\right.$ is the Lebesque number of $\mathcal{V}_{n}$ ).

There exists a sequence $\gamma_{n}<1$ such that $\mathcal{U}_{n}=\tau^{-1}\left[c_{i}-\gamma_{n}, c_{i}+\gamma_{n}\right] \subseteq T\left(M_{i}, \lambda_{n} / 8\right)$. In the contrary a sequence $z_{m} \in \mathcal{U}_{m}=\tau^{-1}\left[c_{i}-1 / m, c_{i}+1 / m\right]$ would exists, such that $\tau\left(z_{m}\right) \longrightarrow c_{i}$ and $z_{m} \notin T\left(M_{i}, \lambda_{n} / 8\right)$. The space is compact so we can suppose that the sequence is convergent i.e. $z_{m} \longrightarrow z$, but from $\tau\left(z_{m}\right) \longrightarrow \tau(z)=c_{i}$ we obtain that $z \in M_{i}$ which is a contradiction with $z_{m} \notin T\left(M_{i}, \lambda_{n} / 8\right), \forall m$.

According to the previous definition of composition of shape morphism we have that $\left[\left(i_{n}\right)\right] \circ\left[\left(f_{n}\right)\right]=$ $\left[\left(i_{n} \circ f_{k_{n}}\right)\right]$ where for the subsequence $k_{n}$ we will impose additional condition, namely to satisfy the following inequality:

$$
t_{k_{n}} \gamma_{n} \geq \max \left\{t_{n}^{+}, t_{n}^{-}\right\}
$$

Now we are ready to define a map

$$
H_{n}:\left(\mathcal{U}^{+} \cup \mathcal{U}^{-}\right) \times I \longrightarrow \mathcal{U}^{+} \cup \mathcal{U}^{-},
$$

which will be a homotopy candidate between $\left(i \circ f_{k_{n}}\right)$ and $\left(1_{\mathcal{u}}\right)$ by:

$$
H_{n}(x, t)=\left\{\begin{array}{lr}
i\left(a\left(x,\left|2 t t_{k_{n}}\left(\tau(x)-c_{i}\right)\right|+t_{k_{n}}(1-2 t)\right)\right), & 0 \leq t \leq \frac{1}{2}, \\
\varphi\left(x, t_{k_{n}}\left(\tau(x)-c_{i}\right)(2-2 t)\right), & \frac{1}{2}<t \leq 1 .
\end{array}\right.
$$

The connection relation is obvious. Let us check how close this map is to continuous:

i) For points $\left(x_{0}, t_{0}\right), x_{0} \in \mathcal{U}, t_{0}>1 / 2$ the map is continuous because the phase map and the Lyapunov function are actually such.

ii) Let $x_{0} \in \operatorname{int} M_{i}, t_{0}<1 / 2$. There exists a neighborhood $W_{0}$ of $x_{0}$ and $W^{0}$ of $t_{0}$ such that $H_{n}(x, t)=$ $\varphi\left(x, t_{k_{n}}(1-2 t)\right), \forall x \in W_{0}, \forall t \in W^{0}$, so the continuity follows.

iii) Let us discuss the points $\left(x_{0}, t_{0}\right), x_{0} \in \operatorname{int} M_{i}, t_{0}=1 / 2$. We shall define the following map $h_{n}: I \longrightarrow \mathbb{R}$ by $h_{n}(t)=\left\{\begin{array}{l}t_{k_{n}}(1-2 t), 0 \leq t \leq 1 / 2, \\ 0, \\ 1 / 2<t \leq 1\end{array}\right.$ which is continuous. There exist neighborhoods $W_{0} \subseteq M_{i}$ of $x_{0}$ and $W^{0}$ of $t_{0}$ such that $H_{n}(x, t)=\varphi\left(x, h_{n}(t)\right)=x, \forall x \in W_{0}, \forall t \in W^{0}$, so we obtain continuity in this case as well.

iv) Consider the points $\left(x_{0}, t_{0}\right), x_{0} \in \partial M_{i}, 0 \leq t_{0}<1 / 2 . H_{n}\left(x_{0}, t_{0}\right)=\varphi\left(x_{0}, t_{k_{n}}\left(1-2 t_{0}\right)\right)$. We choose arbitrary neighborhood $Q$ of this point $\left(H_{n}\left(x_{0}, t_{0}\right)\right)$. There exist neighborhoods $W_{0}$ of $x_{0}$ and $W^{0} \subseteq[0,1 / 2)$ of $t_{0}$ such that $H_{n}\left(W_{0} \cap M_{i}, W^{0}\right) \subseteq Q$. There exists $\varepsilon>0$ such that $T\left(H_{n}\left(x_{0}, t_{0}\right), \varepsilon / 2\right) \subseteq T\left(H_{n}\left(x_{0}, t_{0}\right), \varepsilon\right) \subseteq \mathcal{Q}$. Let us consider the following map $h_{n}(x, t)=\left|2 t t_{k_{n}}\left(\tau(x)-c_{i}\right)\right|+t_{k_{n}}(1-2 t)$ which is continuous. There exist neighborhoods $W_{1}$ of $x_{0}$ and $W^{1}$ of $t_{0}$ such that $\forall x \in\left(\mathcal{U}^{+} \backslash M_{i}\right) \cap W_{1}, \forall t \in W^{1}$ :

$$
d\left(\varphi\left(x, h_{n}(x, t)\right), a\left(x, h_{n}(x, t)\right)\right)<\frac{\varepsilon}{2}, \varphi\left(x, h_{n}(x, t)\right) \in T\left(H_{n}\left(x_{0}, t_{0}\right), \varepsilon / 2\right) .
$$

Analogously, there exist neighborhoods $W_{2}$ of $x_{0}$ and $W^{2}$ of $t_{0}$ such that $\forall x \in\left(\mathcal{U}^{-} \backslash M_{i}\right) \cap W^{2}, \forall t \in W^{2}$ :

$$
d\left(\varphi\left(x,-h_{n}(x, t)\right), a\left(x, h_{n}(x, t)\right)\right)<\frac{\varepsilon}{2}, \varphi\left(x,-h_{n}(x, t)\right) \in T\left(H_{n}\left(x_{0}, t_{0}\right), \varepsilon / 2\right) .
$$

We choose $W_{0} \cap W_{1} \cap W_{2}=W_{\star}$ as a neighborhood of $x_{0}$ and $W^{0} \cap W^{1} \cap W^{2}=W^{\star}$ as a neighborhood of $t_{0}$. Hence $H_{n}\left(W_{\star}, W^{\star}\right) \subseteq Q$, so the continuity is confirmed in this case as well. 
v) Let's consider the case for the points $\left(x_{0}, t_{0}\right)$ such that $x_{0} \in \partial M_{i}, t_{0}=1 / 2$.

$$
H_{n}\left(x_{0}, 1 / 2\right)=a\left(x_{0}, t_{k_{n}}(1-2 \cdot 1 / 2)\right)=x_{0} .
$$

We choose arbitrary neighborhood 2 of $H_{n}\left(x_{0}, 1 / 2\right)$. There exist neighborhoods $W_{0}$ of $x_{0}$ and $W^{0}$ of $t_{0}=1 / 2$ such that $H_{n}\left(W_{0}, W^{0} \cap(1 / 2,1]\right) \subseteq 2$. From the previous case there exist neighborhoods $W_{1}$ of $x_{0}$ and $W^{1}$ of $t_{0}=1 / 2$ such that $H_{n}\left(W_{1}, W^{1} \cap[0,1 / 2]\right) \subseteq 2$. We choose $W_{\star}=W_{0} \cap W_{1}$ as a neighborhood for $x_{0}$ and $W^{\star}=W^{0} \cap W^{1}$ as a neighborhood for $t_{0}=1 / 2$. From the previous discussion we obtain the following inclusion $H_{n}\left(W_{\star}, W^{\star}\right) \subseteq \mathcal{Q}$ and the continuity in these points follows.

vi) Let us consider the points $\left(x_{0}, t_{0}\right), t_{0}<1 / 2$ and such that $\left|\tau\left(x_{0}\right)-c_{i}\right|>\gamma_{n}$. We suppose that $x_{0} \in \mathcal{U}^{+}$. We consider the function $h_{n}: X \times \mathbb{R} \longrightarrow \mathbb{R}$ defined by $h_{n}(x, t)=\left|2 t t_{k_{n}}\left(\tau(x)-c_{i}\right)\right|+t_{k_{n}}(1-2 t)$. Note that $h_{n}(x, t) \geq$ $\max \left\{t_{n}^{+}, t_{n}^{-}\right\}$. Namely, $h_{n}(x, t)>2 t t_{k_{n}} \gamma_{n}+t_{k_{n}}(1-2 t)$ (in a neighborhood of the point $x_{0}$ ) and the right side of the inequality if we treat it as a linear map on the segment $[0,1 / 2]$ it obtains its minimum at $t=1 / 2$ so:

$$
h_{n}(x, t)>t_{k_{n}} \gamma_{n} \geq \max \left\{t_{n}^{+}, t_{n}^{-}\right\}
$$

There exist neighborhoods $W_{0}$ of $x_{0}$ and $W^{0}$ of $t_{0}$ such that

$$
d\left(\varphi\left(x, h_{n}(x, t)\right), a\left(x, h_{n}(x, t)\right)\right) \leq \frac{\lambda_{n}}{8}, \forall x \in W_{0}, \forall t \in W^{0} .
$$

Specially $d\left(\varphi\left(x, h_{n}(x, t)\right), a\left(x_{0}, h_{n}\left(x_{0}, t_{0}\right)\right)\right) \leq \lambda_{n} / 8$. Now, from the continuity of the phase map $\varphi$ and a choice for $\varepsilon<\lambda_{n} / 4$ there exist neighborhoods $W_{1}$ of $x_{0}$ and $W^{1}$ of $t_{0}$ such that

$$
d\left(\varphi\left(x, h_{n}(x, t)\right), \varphi\left(x_{0}, h_{n}\left(x_{0}, t_{0}\right)\right)\right)<\varepsilon, \forall x \in W_{1}, \forall t \in W^{1} .
$$

Let $W_{\star}=W_{0} \cap W_{1}$ and $W^{\star}=W^{0} \cap W^{1}$ are the neighborhoods for $x_{0}$ and $t_{0}$ correspondingly. Note the following inequality:

$$
\begin{gathered}
d\left(a\left(x, h_{n}(x, t), a\left(x_{0}, h_{n}\left(x_{0}, t_{0}\right)\right)\right) \leq d\left(a\left(x, h_{n}(x, t), \varphi\left(x, h_{n}(x, t)\right)\right)\right.\right. \\
+d\left(\varphi\left(x, h_{n}(x, t)\right), \varphi\left(x_{0}, h_{n}\left(x_{0}, t_{0}\right)\right)\right)+d\left(\varphi\left(x_{0}, h_{n}\left(x_{0}, t_{0}\right)\right), a\left(x_{0}, h_{n}\left(x_{0}, t_{0}\right)\right)\right) \\
<\frac{\lambda_{n}}{8}+\frac{\lambda_{n}}{4}+\frac{\lambda_{n}}{8}=\frac{\lambda_{n}}{2}, \quad \forall x \in W_{\star}, \forall t \in W^{\star} .
\end{gathered}
$$

Now, for arbitrary $x_{1}, x_{2} \in W \star$ and $t_{1}, t_{2} \in W^{\star}$, we obtain that:

$$
d\left(a\left(x_{1}, h_{n}\left(x_{1}, t_{1}\right)\right), a\left(x_{2}, h_{n}\left(x_{2}, t_{2}\right)\right)\right)<\lambda_{n}
$$

which is exactly $\mathcal{V}_{n}$-continuity in the point $\left(x_{0}, t_{0}\right)$. Similarly for the points $\left(x_{0}, t_{0}\right), t_{0}<1 / 2,\left|\tau\left(x_{0}\right)-c_{i}\right|>\gamma_{n}$ and $x_{0} \in \mathcal{U}^{-}$.

vii) Now let us consider the points $\left(x_{0}, t_{0}\right), t_{0}<1 / 2,\left|\tau\left(x_{0}\right)-c_{i}\right| \leq \gamma_{n}, x_{0} \in \mathcal{U}^{+} \backslash M_{i}$. Note that $\mathcal{U}_{n}=\tau^{-1}\left(c_{i}-\gamma_{n}-\right.$ $\left.\delta, c_{i}+\gamma_{n}+\delta\right) \subseteq T\left(M_{i}, \lambda_{n} / 8\right)$, for sufficiently small $\delta>0$. Let $h_{n}(x, t)=\left|2 t t_{k_{n}}\left(\tau(x)-c_{i}\right)\right|+t_{k_{n}}(1-2 t)$. From the assumption $x_{0} \in \mathcal{U}_{n}$. We shall prove that the set $\tau^{-1}\left(c_{i}, c_{i}+\gamma_{n}+\delta\right)$ is positively invariant. Namely, if $p \in \tau^{-1}\left(c_{i}, c_{i}+\gamma_{n}+\delta\right)$ then for arbitrary $t>0$ we have

$$
c_{i}<\tau(\varphi(p, t))<\tau(p)<c_{i}+\gamma_{n}+\delta
$$

We shell explain only the first inequality $c_{i}<\tau(\varphi(p, t))$. In the contrary $c_{i} \geq \tau(\varphi(p, t))$ then $p \in M_{i}$ which is a contradiction. Now, from $x_{0} \in \tau^{-1}\left(c_{i}, c_{i}+\gamma_{n}+\delta\right)$ there exists a neighborhood $W_{0}$ of $x_{0}$ such that $W_{0} \subseteq$ $\tau^{-1}\left(c_{i}, c_{i}+\gamma_{n}+\delta\right)$. Let $W^{0}$ is a neighborhood of $t_{0}$ such that $W^{0} \subseteq[0,1 / 2)$. Then

$$
d\left(\varphi\left(x, h_{n}(x, t)\right), a\left(x, h_{n}(x, t)\right)\right)<\frac{\lambda_{n}}{8}, \forall x \in W_{0}, \forall t \in W^{0} .
$$

Specially, for $x=x_{0}$ and $t=t_{0}$ we have that

$$
d\left(\varphi\left(x_{0}, h_{n}\left(x_{0}, t_{0}\right)\right), a\left(x_{0}, h_{n}\left(x_{0}, t_{0}\right)\right)\right)<\frac{\lambda_{n}}{8} .
$$


Now, from the continuity of the phase map for a choice of $\varepsilon<\lambda_{n} / 4$ there exist neighborhoods $W_{1}$ of $x_{0}$ and $W^{1}$ of $t_{0}$ such that

$$
d\left(\varphi\left(x, h_{n}(x, t)\right), \varphi\left(x_{0}, h_{n}\left(x_{0}, t_{0}\right)\right)\right)<\varepsilon, \forall x \in W_{1}, \forall t \in W^{1} .
$$

Again, for a choice $W_{\star}=W_{0} \cap W_{1}$ as a neighborhood of $x_{0}$ and $W^{\star}=W^{0} \cap W^{1}$ as a neighborhood of $t_{0}$ we obtain

$$
\begin{gathered}
d\left(a\left(x, h_{n}(x, t)\right), a\left(x_{0}, h_{n}\left(x_{0}, t_{0}\right)\right)\right) \leq d\left(a\left(x, h_{n}(x, t)\right), \varphi\left(x, h_{n}(x, t)\right)\right) \\
+d\left(\varphi\left(x, h_{n}(x, t)\right), \varphi\left(x_{0}, h_{n}\left(x_{0}, t_{0}\right)\right)\right)+d\left(\varphi\left(x_{0}, h_{n}\left(x_{0}, t_{0}\right)\right), a\left(x_{0}, h_{n}\left(x_{0}, t_{0}\right)\right)\right) \\
<\frac{\lambda_{n}}{8}+\frac{\lambda_{n}}{4}+\frac{\lambda_{n}}{8}=\frac{\lambda_{n}}{2}, \quad \forall x \in W_{\star}, \forall t \in W^{\star},
\end{gathered}
$$

which means that we have $V_{n}$-continuous in this point as well. The discussion about the points $\left(x_{0}, t_{0}\right), t_{0}<$ $1 / 2,\left|\tau\left(x_{0}\right)-c_{i}\right| \leq \gamma_{n}, x_{0} \in \mathcal{U}^{-} \backslash M_{i}$ is similar.

viii)The points $\left(x_{0}, 1 / 2\right), x_{0} \in \mathcal{U} \backslash M_{i}$ are remained for discussion. First let us suppose that $x_{0} \in \mathcal{U}^{+}$. We shell make another assumption, namely $\tau\left(x_{0}\right)<\alpha_{n}$. We shell prove that $\tau(x)<\alpha_{n}, x \in \mathcal{U}^{+}$implies $x \in T\left(M_{i}, \lambda_{n} / 8\right)$. In the contrary there exists $t \geq 0$ such that $\varphi(x, t) \in S\left(M_{i}, \lambda_{n} / 8\right)$, because the trajectory segments are compact connected sets, which means that $\tau(x) \geq \tau(\varphi(x, t)) \geq \alpha_{n}$. This is a contradiction. There exists a neighborhood $W_{0} \subseteq \mathcal{U}^{+} \backslash M_{i}$ of $x_{0}$ such that $\tau(x)<\alpha_{n}$ for all $x \in W_{0}$. Note that $H_{n}\left(x_{0}, 1 / 2\right)=a\left(x_{0}, t_{k_{n}}\left(\tau\left(x_{0}\right)-c_{i}\right)\right)$. Let $\varepsilon=\lambda_{n} / 4$. There exist neighborhoods $W_{1}$ of $x_{0}$ and $W^{1}$ of $1 / 2$ such that:

$$
d\left(\varphi\left(x, 2 t t_{k_{n}}\left(\tau(x)-c_{i}\right)+t_{k_{n}}(1-2 t)\right), \varphi\left(x_{0}, t_{k_{n}}\left(\tau\left(x_{0}\right)-c_{i}\right)\right)\right)<\frac{\lambda_{n}}{4}, \forall x \in W_{1}, \forall t \in W^{1} .
$$

Let $W_{\star}=W_{0} \cap W_{1}$ and $W^{\star}=W^{1}$. Also, let $h_{n}(x, t)=2 t t_{k_{n}}\left(\tau(x)-c_{i}\right)+t_{k_{n}}(1-2 t)$. Note that, $\forall x \in W_{\star}$ and $t \in W^{\star} \cap[0,1 / 2]$, we have that $\alpha_{n}>\tau(x) \geq \tau\left(\varphi\left(x, h_{n}(x, t)\right)\right)$ which means that $\varphi\left(x, h_{n}(x, t)\right) \in T\left(M_{i}, \lambda_{n} / 8\right)$. Now, for arbitrary $x \in W_{\star}$ and $t \in W^{\star} \cap[0,1 / 2]$ we have the following estimate:

$$
\begin{gathered}
d\left(a\left(x, h_{n}(x, t)\right), a\left(x_{0}, h_{n}\left(x_{0}, 1 / 2\right)\right)\right) \leq d\left(a\left(x, h_{n}(x, t)\right), \varphi\left(x, h_{n}(x, t)\right)\right) \\
+d\left(\varphi\left(x, h_{n}(x, t)\right), \varphi\left(x_{0}, h_{n}\left(x_{0}, 1 / 2\right)\right)\right)+d\left(\varphi\left(x_{0}, h_{n}\left(x_{0}, 1 / 2\right)\right), a\left(x_{0}, h_{n}\left(x_{0}, 1 / 2\right)\right)\right) \\
<\frac{\lambda_{n}}{8}+\frac{\lambda_{n}}{4}+\frac{\lambda_{n}}{8}=\frac{\lambda_{n}}{2} .
\end{gathered}
$$

There exists an element $V_{n}$ of $\mathcal{V}_{n}$ such that $H_{n}\left(W_{\star}, W^{\star} \cap[0,1 / 2]\right) \subseteq V_{n}$ is valid. For $g_{n}(x, t)=t_{k_{n}}\left(\tau(x)-c_{i}\right)(2-$ $2 t)$ and $\varepsilon=3 \lambda_{n} / 16$ there exist neighborhoods $W_{2}$ of $x_{0}$ and $W^{2}$ of $1 / 2$ such that $d\left(\varphi\left(x, g_{n}(x, t)\right), \varphi\left(x, g_{n}(x, 1 / 2)\right)\right)<$ $3 \lambda_{n} / 16$. Analogously there exists neighborhood $W_{3}$ of $x_{0}$ such that: $d\left(\varphi\left(x, g_{n}(x, 1 / 2)\right), \varphi\left(x_{0}, g_{n}\left(x_{0}, 1 / 2\right)\right)\right)<$ $\frac{3 \lambda_{n}}{16}$. Let $W_{\star \star}=W_{0} \cap W_{2} \cap W_{3}$ is a neighborhood of $x_{0}$ and $W_{\star \star}=W^{2}$ a neighborhood of $1 / 2$. We obtain the following estimates:

$$
\begin{gathered}
d\left(\varphi\left(x, g_{n}(x, t)\right), a\left(x_{0}, g_{n}\left(x_{0}, 1 / 2\right)\right)\right) \leq d\left(\varphi\left(x, g_{n}(x, t)\right), \varphi\left(x, g_{n}(x, 1 / 2)\right)\right) \\
+d\left(\varphi\left(x, g_{n}(x, 1 / 2)\right), \varphi\left(x_{0}, g_{n}\left(x_{0}, 1 / 2\right)\right)\right)+d\left(\varphi\left(x_{0}, g_{n}\left(x_{0}, 1 / 2\right)\right), a\left(x_{0}, g_{n}\left(x_{0}, 1 / 2\right)\right)\right) \\
<\frac{3 \lambda_{n}}{16}+\frac{3 \lambda_{n}}{16}+\frac{\lambda_{n}}{8}=\frac{\lambda_{n}}{2} .
\end{gathered}
$$

This means that there exists $V_{n}^{\star} \in \mathcal{V}_{n}$ such that $H_{n}\left(W_{\star \star}, W^{\star \star} \cap(1 / 2,1]\right) \subseteq V_{n}^{\star}$. Also, let us note that the following inclusion $H_{n}\left(W_{\star \star}, W^{\star \star} \cap[1 / 2,1]\right) \subseteq V_{n}^{\star}$. Namely, the neighborhood $W_{\star \star}$ can be chosen sufficiently small such that:

$$
d\left(\varphi\left(x_{1}, t_{k_{n}}\left(\tau\left(x_{1}\right)-c_{i}\right)\right), \varphi\left(x_{2}, t_{k_{n}}\left(\tau\left(x_{2}\right)-c_{i}\right)\right)\right)<\frac{\lambda_{n}}{16}, \forall x_{1}, x_{2} \in W_{\star \star} .
$$

Having this in mind for the points $\left(x_{1}, 1 / 2\right),\left(x_{2}, 1 / 2\right), x_{1}, x_{2} \in W_{\star \star}$ we have that the following holds:

$$
d\left(H_{n}\left(x_{1}, 1 / 2\right), H_{n}\left(x_{2}, 1 / 2\right)\right) \leq d\left(H_{n}\left(x_{1}, 1 / 2\right), \varphi\left(x_{1}, g_{n}\left(x_{1}, 1 / 2\right)\right)\right.
$$




$$
\begin{gathered}
+d\left(\varphi \left(x_{1}, g_{n}\left(x_{1}, 1 / 2\right), \varphi\left(x_{2}, g_{n}\left(x_{2}, 1 / 2\right)\right)+d\left(\varphi\left(x_{2}, g_{n}\left(x_{2}, 1 / 2\right)\right), H_{n}\left(x_{2}, 1 / 2\right)\right)\right.\right. \\
<\frac{\lambda_{n}}{8}+\frac{\lambda_{n}}{16}+\frac{\lambda_{n}}{8}<\lambda_{n} .
\end{gathered}
$$

Similarly, for the points $\left(x_{1}, 1 / 2\right),\left(x_{2}, t_{2}\right), x_{1}, x_{2} \in W_{\star \star}, t_{2}>1 / 2$ we have :

$$
\begin{gathered}
d\left(H_{n}\left(x_{2}, t_{2}\right), H_{n}\left(x_{1}, 1 / 2\right)\right) \leq d\left(H_{n}\left(x_{2}, t_{2}\right), \varphi\left(x_{2}, g_{n}\left(x_{2}, 1 / 2\right)\right)\right) \\
+d\left(\varphi\left(x_{2}, g_{n}\left(x_{2}, 1 / 2\right), a\left(x_{2}, g_{n}\left(x_{2}, 1 / 2\right)\right)\right)+d\left(a\left(x_{2}, g_{n}\left(x_{2}, 1 / 2\right)\right), a\left(x_{1}, g_{n}\left(x_{1}, 1 / 2\right)\right)\right)\right. \\
<\frac{3 \lambda_{n}}{16}+\frac{\lambda_{n}}{8}+\frac{2 \lambda_{n}}{8}+\frac{\lambda_{n}}{16}<\lambda_{n} .
\end{gathered}
$$

Hence, $V_{n}^{\star} \cap V_{n} \neq \emptyset$. But this implies that we have $\operatorname{st}\left(\mathcal{V}_{n}\right)$-continuity in this points.

Now, we shall discuss the points $\tau\left(x_{0}\right) \geq \alpha_{n}$. From the inequality $\tau\left(x_{0}\right)-c_{i} \geq \alpha_{n}-c_{i}$ we obtain that

$$
\left(\tau\left(x_{0}\right)-c_{i}\right) t_{k_{n}} \geq\left(\tau\left(x_{0}\right)-c_{i}\right) t_{n} \geq \frac{t_{n}^{+}}{\alpha_{n}-c_{i}} \cdot\left(\tau\left(x_{0}\right)-c_{i}\right) \geq t_{n}^{+},
$$

but $\varphi\left(u, t_{n}^{+}\right) \in T\left(M_{i}, \lambda_{n} / 8\right), \forall u \in \mathcal{U}^{+}$we have $\varphi\left(x_{0}, t_{k_{n}}\left(\tau\left(x_{0}\right)-c_{i}\right)\right) \in T\left(M_{i}, \lambda_{n} / 8\right)$.

Now, from the inequality $t_{n} \geq t_{n}^{+}$we obtain that:

$$
\begin{gathered}
h_{n}(x, t)=2 t t_{k_{n}}\left(\tau(x)-c_{i}\right)+t_{k_{n}}(1-2 t)>2 t t_{k_{n}}\left(\alpha_{n}-c_{i}\right)+t_{k_{n}}(1-2 t) \\
\geq 2 t t_{n}\left(\alpha_{n}-c_{i}\right)+t_{n}(1-2 t) \geq 2 t t_{n}^{+}+t_{n}^{+}(1-2 t)=t_{n}^{+}, \forall t \in[0,1 / 2]
\end{gathered}
$$

, which implies that $\varphi\left(x, 2 t t_{k_{n}}\left(\tau(x)-c_{i}\right)+t_{k_{n}}(1-2 t)\right) \in T\left(M_{i}, \lambda_{n} / 8\right)$. So we have st $\left(\mathcal{V}_{n}\right)$-continuity in this points as well.

Now we shall assume that $x_{0} \in \mathcal{U}^{-} \backslash M_{i}$, which is the remaining case. We make additional assumption $\tau\left(x_{0}\right)>$ $\beta_{n}$. We shall prove that $\tau(x)>\beta_{n}, x \in \mathcal{U}^{-}$implies that $x \in T\left(M_{i}, \lambda_{n} / 8\right)$. On the contrary, there exists $t>0$ such that $\tau(\varphi(x,-t)) \in S\left(M_{i}, \lambda_{n} / 8\right)$. But, this would imply that

$$
\beta_{n}<\tau(x) \leq \tau(\varphi(x,-t)) \leq \beta_{n},
$$

which is a contradiction. Now, from the inequality:

$$
\tau\left(\varphi\left(x_{0}, t_{k_{n}}\left(\tau\left(x_{0}\right)-c_{i}\right)\right)\right) \geq \tau\left(x_{0}\right)>\beta_{n},
$$

we have that $\varphi\left(x_{0}, t_{k_{n}}\left(\tau\left(x_{0}\right)-c_{i}\right)\right) \in T\left(M_{i}, \lambda_{n} / 8\right)$. According to the previous discussion, similarly we obtain $\operatorname{st}\left(\mathcal{V}_{n}\right)$-continuity in this points. It remains the case when $\tau\left(x_{0}\right) \leq \beta_{n}$. Note the inequality $\tau\left(x_{0}\right)-c_{i} \leq \beta_{n}-c_{i}$ in which all the values are negative. This implies that

$$
\left(\tau\left(x_{0}\right)-c_{i}\right) t_{k_{n}} \leq\left(\beta_{n}-c_{i}\right) t_{n}=\left(\beta_{n}-c_{i}\right) \cdot \frac{t_{n}^{-}}{c_{i}-\beta_{n}}=-t_{n}^{-} .
$$

Again, from $\varphi\left(x_{0},-t_{n}^{-}\right) \in T\left(M_{i}, \lambda_{n} / 8\right)$ we have $\varphi\left(x_{0}, t_{k_{n}}\left(\tau\left(x_{0}\right)-c_{i}\right)\right) \in T\left(M_{i}, \lambda_{n} / 8\right)$, which implies that we have $\operatorname{st}\left(\mathcal{V}_{n}\right)$-continuity in this points as well.

The proof is complete.

Let us note that the condition imposed on the Lyapunov function is essential in theorem 3.1 as shown in the following example:

\section{Example 3.1.}

Consider a dynamical system defined in the cylinder $D \times I$, where $D$ stands for the unit disk. The points in the Hawaiian earring $H=\bigcup_{n=1}^{\infty} S((1 / 2 n, 0,1 / 2), 1 / 2 n)$ are stationary points. All the points in $D \times\{0,1\}$ are also stationary. The trajectories of the rest of the points are vertical straight lines joining two stationary points. 
The Hawaiian earring is a Morse set which is not a shape retract of any of its neighborhoods. This shows that theorem 3.1 does not hold for general Lyapunov functions.

Now, we are ready to consider the shape of chain recurrent set $\mathcal{C R}(\varphi)$.

We shall use the following theorem of Conley [14].

Theorem 3.2. Let $(X, d)$ be a compact metric space with a flow $\varphi$. Then there exists a unique family $\mathcal{M}=\left\{M_{j} \mid j \in\right.$ $J\}$ of disjoint, compact and invariant sets which admits Lyapunov function and is maximal with this property. The set $\cup_{j \in J} M_{j}$ is actually $\mathcal{e R}(\varphi)$, which is compact and $M_{j}$ are its connected components.

Corollary 3.1. Arbitrary chain recurrent set with finitely many connected components satisfying the conditions of the previous theorem 3.1 admits compact neighborhood $U$ with the same shape as $\mathcal{C R}(\varphi)$, i.e. $\operatorname{Sh}(\mathcal{U})=$ $\operatorname{Sh}(\mathrm{CR}(\varphi))$.

Proof. From the previous theorem of Conley, the connected components of $\mathcal{C R}(\varphi), \mathcal{M}=\left\{\mathcal{C R}_{j}(\varphi) \mid j \in J\right\}$, form a Morse decomposition. According to the previously proven theorem, each of this components admits a compact neighborhood $\mathcal{U}_{i}$ with the same shape as $\mathcal{C R}_{i}(\varphi)$, i.e. $\operatorname{Sh}\left(\mathcal{U}_{i}\right)=\operatorname{Sh}\left(\mathcal{e} \mathcal{R}_{i}(\varphi)\right)$ and such that is disjoint from the others. Now, if we choose $\mathcal{U}=\cup_{i=1}^{n} \mathfrak{U}_{i}$, we get that

$$
\operatorname{Sh}(\mathcal{U})=\operatorname{Sh}\left(\cup_{i=1}^{n} \mathcal{U}_{i}\right)=\operatorname{Sh}\left(\cup_{i=1}^{n} \mathcal{C R}_{i}(\varphi)\right)=\operatorname{Sh}(\mathcal{E R}(\varphi)) .
$$

In the section that follows we shall verify this result on a couple of systems of differential equations by investigating the symbolic image (an oriented graph) with respect to a given covering. We used the algorithm suggested by Osipenko in [3] to get a sequence of embedded neighborhoods which converges to the chain recurrent set by writing a programming code in Mathematica.

\section{Symbolical analysis of the Chain Recurrent Set}

The theoretical background of the symbolical images and the constructive methods which are applied on them were described in detail by Osipenko in [17]. The main idea is construction of a directed graph which represents the structure of the state space for the investigated dynamical system. This graph is called the symbolical image of the focused system and can be seen as approximation of the system flow. Valuable information about the global structure of the system may come from the analysis of this symbolic image. From the computation point of view the usage of such a graph has the advantage that once it is constructed, all investigations are matters of graph analysis which provides an opportunity among other things in it's pallet an algorithmic way of thinking.

Hence basic operations on the symbolical image gives an opportunity to obtain a sequence of neighborhoods which localize invariant sets, or in our case of interest, the chain recurrent set. Numerical computations are performed for several dynamical systems in order to verify our theoretical result.

Let us consider a discrete dynamical system governed by a homeomorphism $f$ defined on a compact manifold $M$.

We use the following algorithm from [3] based on which a programming code in "Mathematica" is successfully obtained and tested on couple of systems of differential equations:

Step1. Starting with an initial covering $\mathcal{C}$, the symbolical image $G=(T, R)$ of the map $f$ is found. The cells of the initial covering may have arbitrarily large diameter $d_{0}$.

Step2. The recurrent vertices $i_{k}$ of the graph $G$ are recognized. Using the recurrent vertices, a closed neighborhood $\mathcal{P}=\bigcup_{i_{k}} M\left(i_{k}\right)$ of the chain recurrent set $\mathcal{e} \mathcal{R}(f)$ is obtained.

Step3. The cells corresponding to the recurrent vertices i.e. $\left\{M\left(i_{k}\right) \mid i_{k}\right.$ is recurrent $\}$ are partitioned. For example, the largest diameter of the cells may be divided with 2 . Thus the new covering is defined. 
Step4. The symbolic image $G=(T, R)$ is constructed for the new covering. It should be noted that the new symbolic image may be constructed on the set $\mathcal{P}=\bigcup_{i_{k}} M\left(i_{k}\right)$. In other words, the cells corresponding to non recurrent vertices do not participate in the construction of the new covering and the new symbolic image.

Step5. Go back to step2.

Repeating this partitioning process we obtain a sequence of neighborhoods $\mathcal{P}_{0}, \mathcal{P}_{1}, \mathcal{P}_{2}, \ldots$ of the chain recurrent set $\mathcal{C R}(f)$.

Before giving some examples of system of differential equations we shall discuss the transition from discrete to continuous phase maps. Namely, the idea is quite simple, discretization of systems continuous in time. Let a system of differential equations be given by $x(t)=F(x(t))$. What we need is some kind of mapping which transforms an orbit continuous in time into one discrete in time. A shift operator along trajectories is needed. Such a mapping has the form $f(x)=\varphi(x, t)$, with $\varphi(x, 0)=x$. It can be calculated by solving the equation. If we fix a value for $t=t_{0}$ we obtain the required discretization.

\section{Example 4.1.}

Consider the following system of differential equations:

$$
\begin{aligned}
& \dot{x}=-y+x\left(1-x^{2}-y^{2}\right) \\
& \dot{y}=x+y\left(1-x^{2}-y^{2}\right) .
\end{aligned}
$$

In polar coordinates we have:

$$
\begin{aligned}
& \dot{r}=r\left(1-r^{2}\right) \\
& \dot{\theta}=1
\end{aligned}
$$

with initial values $r(0)=r_{0}$ and $\theta(0)=\theta_{0}$. The first equation can be solved either as a Bernoulli equation or as a separable differential equation. The solution is given by:

$$
r\left(t, r_{0}\right)=\left(1+\left(\frac{1}{r_{0}^{2}}-1\right) e^{-2 t}\right)^{\frac{-1}{2}} \text { and } \theta\left(t, \theta_{0}\right)=t+\theta_{0}
$$

Now we easily obtain the required discretization by choosing a value for $t=10$ for example. (The value of $t$ is important for practical purposes above all because it determines the speed with which the non recurrent cells are being erased but only user experience and heuristic testing can lead to the most proper setting of $t$ ). We input this map in the programming code and obtain the following neighborhoods of $\mathcal{C R}(f)$ :

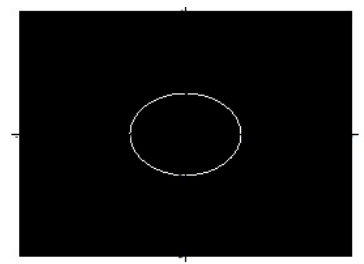

Figure 1: first iteration 


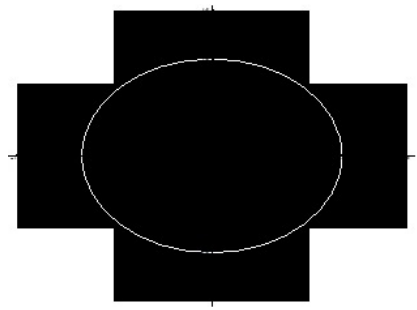

Figure 2: second iteration

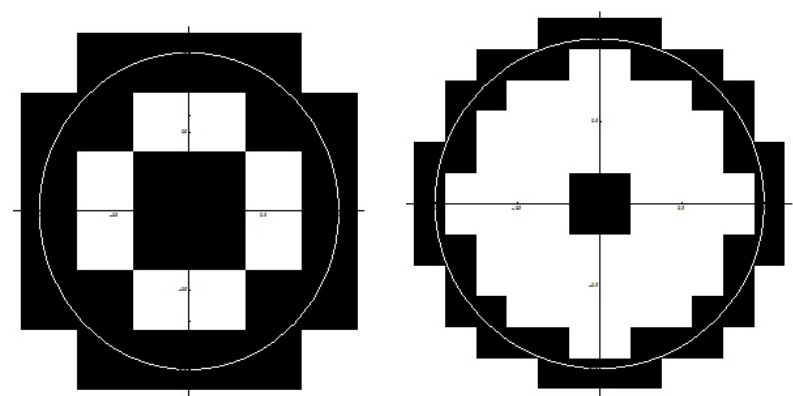

Figure 3: third iteration

fourth iteration

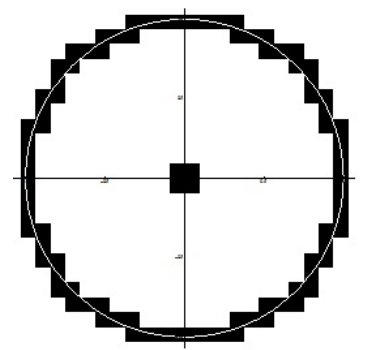

Figure 4: fifth iteration

Consider the following system of differential equations:

$$
\begin{aligned}
& \dot{x}=y \\
& \dot{y}=-x+y \cos (x) .
\end{aligned}
$$

In this system we cannot obtain our discretization map like in the previous example by integrating the system, but using the built in function NDSOLVE in the programming package "Mathematica" we obtain approximation of it. 
These are the results:

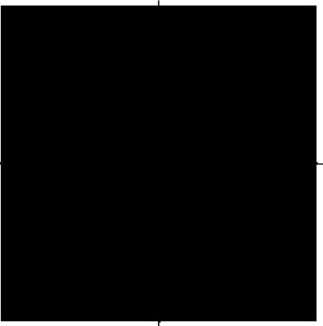

Figure 5: first iteration
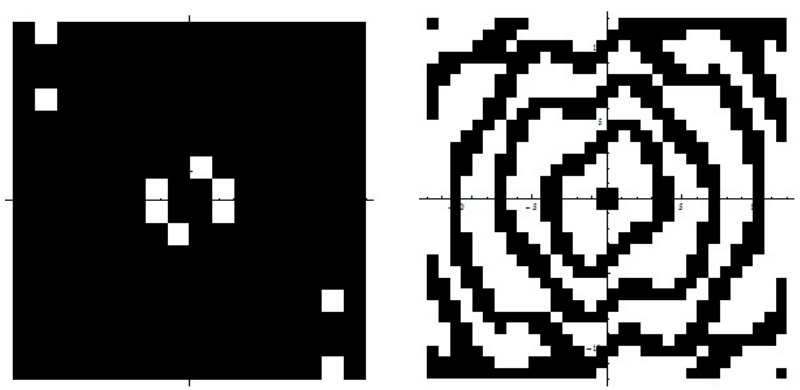

Figure 6: second iteration

third iteration

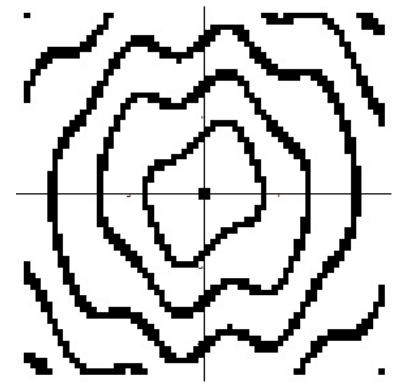

Figure 7: fourth iteration

Let us consider the following system of differential equations in a polar form:

$$
\begin{aligned}
& \dot{r}=r^{2} \sin \left(\frac{10}{r}\right) \\
& \dot{\theta}=1
\end{aligned}
$$

Initially we isolate the point $(0,0)$ by a square box. 
Here are the results:

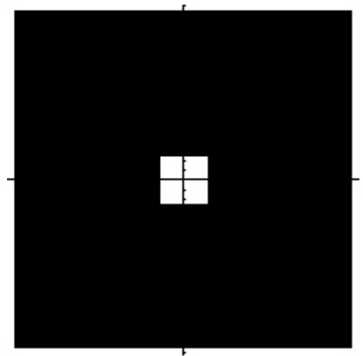

Figure 8: first iteration
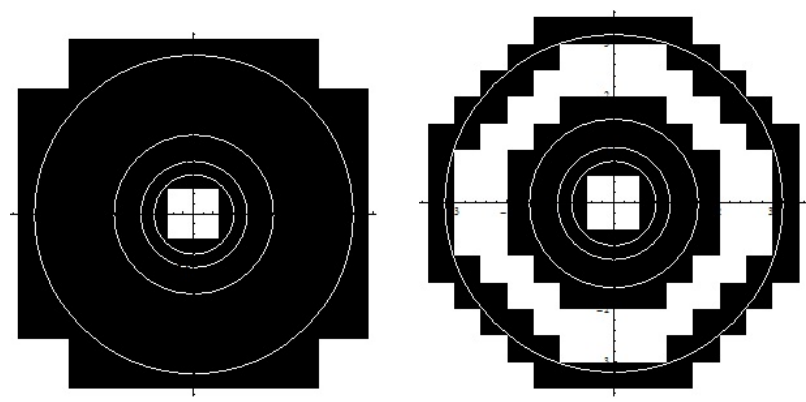

Figure 9: second iteration

third iteration

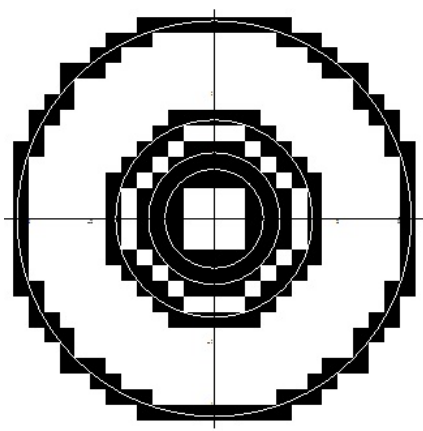

Figure 10: fourth iteration

A computer program realizing the algorithm described above has been made at St.Cyril and Methodius University, Institute for Mathematics, by I. Jovceski and the author.

\section{References}

[1] N. Shekutkovski, Intrinsic definition of strong shape for compact metric spaces, Topology Proceedings, 39 (2012), 27-39.

[2] N. Shekutkovski, M. Shoptrajanov, Intrinsic Shape of the Chain recurrent set, Topology and its Applications, 202 (2016), 117-126. 
[3] G. Osipenko, Symbolical analysis of the chain recurrent trajectories of dynamical systems, Differential Equations and Control Processes, (1998), No.4.

[4] H. M. Hastings, A higher dimensional Poincare- Bendixon theorem, Glasnik Matematicki, 34 (1979), $263-268$.

[5] B. A. Bogatyi, V. I. Gutsu, On the structure of attracting compacta, Differentsial'nye Uravneniya, 25 (1989), $907-909$.

[6] J. B. Gunther, J. Segal, Every attractor of a flow on a manifold has the shape of a finite polyhedron, Proc. AMS, 119 (1993), 321-329.

[7] J. M. Sanjurjo, On the structure of uniform attractors, J.Math. Anal. Appl, 192 (1995), 519-528.

[8] I. Rodnianski, L. Kapitanski, Shape and Morse theory of attractors, Communications On Pure and Applied Mathematics, 53 (2000), no.2, 218-242.

[9] J. M. Sanjurjo, A non continuous description of the shape category of compacta, Quart.J.Math.Oxford, 40 (1989), $351-359$.

[10] M. Shoptrajanov,N. Shekutkovski, Characterization of topologically tranzitive atrractors for analitic plane flows, Filomat, 29:1 (2015), 89-97.

[11] C.C. Conley, The gradient structure of the flow, I.Ergodic Th.Dynam. Systems, 8 (1988), 11-26.

[12] J. E. Felt, $\varepsilon$-continuity and shape, Procedings of the American Mathematical Society, 46 (1974), no.3, 426-430.

[13] C. Zuo, X. Wang, Attractors and Quasi-Attractors of a Flow, J.Appl.Math and Computing, Vol.23 (2007), no.1-2, 411-417.

[14] C.C. Conley, Isolated invariant sets and the Morse index, AMS Providence R.I., 38 (1978), 234-240.

[15] S. Gabites, Dynamical systems and shape, Revista de la Real Academia de Ciencias Exactas, Fizicas y Naturales. Serie A, Matematicas, 102 Vol. 102 (1) (2008), 127-159.

[16] J. Keesling, Locally compact full homeomorphism group are zero-dimensional, Proc. Amer.Math Soc. 29 (1971), $390-396$.

[17] G. Osipenko, Dynamical systems, graphs and algorithms, Springer-Verlag, New York, (2000). 